\title{
GASTRO-ESOPHAGEAL REFLUX DISEASE AND ITS IMPACT ON TRACHEO-ESOPHAGEAL SPEAKING VALVE REHABILITATION AFTER TOTAL LARYNGECTOMY
}

\author{
A. Nicolaescu 1,4, Ș. Berteșteanu ${ }^{2,4}$, R. Grigore ${ }^{2,4}$, B. Popescu ${ }^{2,4}$, R. Hainăroșie ${ }^{3,4}$, V. Zainea ${ }^{3,4}$ \\ 1'Prof. Dr. Dimitrie Gerota” Emergency Hospital, Bucharest - ENT Compartment \\ ${ }^{2}$ Otorhinolaryngology Department - „Colțea” Clinical Hospital, Bucharest \\ ${ }^{3}$ Otorhinolaryngology Department - „Dorin Hociotă” Institute of Phono-Audiology and ENT Functional Surgery, \\ Bucharest \\ 4"Carol Davila" University of Medicine and Pharmacy, Bucharest, Romania \\ Corresponding author: Nicolaescu Alexandru \\ Phone no.: +40213037080 int. 28176 \\ E-mail: alexandrunicolaescu@ymail.com
}

\begin{abstract}
Gastro-esophageal reflux disease (GERD) is a pathological entity in which the mixture of gastric contents (with low $\mathrm{pH}$ because of the high content of $\mathrm{HCl}$ acid) and biliary juice (rich in proteolytic enzymes like pepsin and trypsin) causes lesions on the mucosa lining the esophagus. A higher prevalence of GERD was discovered in patients who had a total laryngectomy. Concerning laryngectomies with tracheo-esophageal speaking valves, it has been demonstrated that GERD is an independent risk factor for failure of this method of speech rehabilitation. The authors performed an analysis of English language articles found following a search of the major medical scientific databases (NCBI®, EMBASE $\AA$, Cochrane $\AA)$ containing the following keyword string: vocal prosthesis, total laryngectomy, GERD, acid reflux, tracheo-esophageal fistula, failure. Studies showed that exposure to the protelolytic enzymes from the reflux juice increases the diameter of the tracheo-esophageal fistula and permits local granulation tissue to form. Progressively, the increasing fistula diameter leads to complications ranging from microaspiration of liquids and saliva to speaking valve expulsion or ingestion and temporary incapacity of oral feeding. Medical therapy in the postoperative period as well as long-term (6 months or more) correction of GERD (PPI therapy, H2 blockers, prokinetics) is also demonstrated to reduce the complication rate in this category of patients. GERD has a high prevalence in the total laryngectomy population of patients and it has multiple etiologic factors. In patients with tracheoesophageal vocal prostheses, GERD causes an increase in the fistula diameter - with important consequences that affect the patient's quality of life - his ability to phonate and his ability to swallow. These complications come with added costs because of the need to more frequently change the vocal prostheses with ones increasing in diameter, as well as multiple hospitalizations (even surgery to recalibrate or close the tracheo-esophageal fistula). Recognizing and treating this condition therefore decreases the risk of complications following vocal prosthesis speech rehabilitation.
\end{abstract}

Keywords: total laryngectom, voice prosthesi, GERD, tracheo-esophageal fistula 


\section{Introduction}

Gastro-esophageal acid reflux disease (GERD) is a complex pathologic condition defined recently in 2006 by the Montreal working group as the disease in which gastric acid reflux causes symptoms or/and complications [1]. Taking into consideration that the typical symptom of GERD, heartburn, is manifest in a large percentage of individuals occasionally without any other associated signs or symptoms, the aforementioned working group decided that for the diagnosis of GERD the patient needs to have constant manifestations or the examining physician has to find one or more complications related to acid reflux (esophagitis, etc.) [1].

Such manifestations related to GERD are chronic cough, chronic laryngitis, non-allergic asthma and dental erosion syndrome. Seeing the high prevalence of GERD in the Western industrialized countries (as much as $40 \%$ according to some authors) [2],[3] we can conclude that GERD is one of the pre-eminent factors responsible for laryngeal pathology, along with smoking, HPV infection and vocal misuse. There are some authors that propose GERD to be listed as an independent risk factor for neoplasia [4][5].

\section{Prevalence of GERD in laryngectomies}

Studies investigating the frequency of postoperative tracheo-esophageal and pharyngocutaneous fistula formation after total laryngectomy found a higher prevalence of GERD in the group of patients which developed a fistula. A direct association was made by Conrad F. Smit and his team who published a study in which they used $24 \mathrm{~h} \mathrm{pH}$-probe monitoring at the level of the upper esophageal sphincter in eleven patients that had a total laryngectomy performed and found pathologic $\mathrm{pH}$ levels in 9 out of the 11 patients [6].

LeBlanc et. al., took this hypothesis further and performed $24 \mathrm{~h} \mathrm{pH}$ monitoring before and after surgery in a group of 10 patients that underwent total laryngectomy. Postoperatively the Ryan score (which is compiled on the number of times the $\mathrm{pH}$ drops under 5.5 in upright as well as lying down positions, the total time the $\mathrm{pH}$ is under 5.5 and the percentage of this time from the total measurement time) doubled [7].

\section{Etiopathology of acid reflux following total laryngectomy}

The reasoning behind this high prevalence of GERD associated with total laryngectomy is the modification of the upper esophageal sphincter. This is done either by direct miotomy of the crico-pharyngeus muscle or by injuring the nervous pharyngeal plexus during the laryngectomy [6],[7]. Another element that serves as protection against acid reflux is the salivary excretion that acts as a clearance mechanism. After total laryngectomy most patients undergo IMRT and adjuvant chemotherapy - that further reduces salivary excretion.

The effect of acid reflux on the pharyngeal and esophageal epithelium is demonstrated by a series of studies on animal and human subjects and is the most severe when $\mathrm{pH}$ levels drop under 1.6. As much as the acidity levels, harmful for the epithelium is the association of pepsin (found in the gastric juice) with the enzymes found in the biliary juice (trypsin, taurocholate, lysolecithin). Pathologic studies found that damage is dose-dependent and progressive, from superficial ulceration, submucous hemorrhage, transmural inflammation to necrosis [8].

\section{Materials and method}

The authors performed an analysis of English language articles found following a search of the major medical scientific databases $\left(\mathrm{NCBI}{ }^{\circledR}\right.$, EMBASE®, Cochrane $®)$ containing the following keyword string: vocal prosthesis AND total laryngectomy OR tracheoesophageal fistula, each associated with GERD OR acid reflux. Read and cited with priority were more recent articles, after the year 2010.

\section{Results}

One of the first cases published by Starling et. al. in 1979 was of a laryngectomy which used esophageal voice to communicate and 
developed severe GERD symptoms as well as a diminishing capacity for esophageal speech. The authors performed a Nissen fundo-plication on the patient - with significant improvement of the symptoms as well as the communication capability [9].

Building on this hypothesis, Mathis et.al. published a paper comparing two groups of laryngectomies that used esophageal voice to communicate. The groups were divided by their ability to effectively use this method in good and adequate ability and the ones that had difficulties in communication. After measuring the pressure in the region of the lower esophageal sphincter as well as the $\mathrm{pH}$ level, the authors concluded that the group of patients with difficulties in using esophageal speech had higher acidity levels and lower closure pressure of the sphincter [10].

Prof. Maier [11] emitted the hypothesis that by using the recommended medical treatment of GERD, one could improve the outcome of voice rehabilitation after total laryngectomy.

More recent studies, like the ones by Seikaly [12], Echegaray [13] and Mannelli [14] demonstrated on significant patient groups that postoperative anti-reflux medication (H2blockers and metoclopramide) can reduce the incidence of pharyngo-cutaneous and tracheoesophageal fistula formation and therefore reduce hospital stay and improve outcome.

Regarding the technique of vocal rehabilitation with tracheo-esophageal implant, the possible complications can be divided into complications related to the malfunction of the device and complications related to the tracheoesophageal shunt. According to the authors, fistula enlargement occurs in almost one out of three patients (13-35\%) [15][16][17].

Lorenz further divides enlargement of the tracheo-esophageal fistula in the atrophic type and the septic-necrotic type (Figure 1) [18]. Septic-necrotic enlargement of the fistula is due to mechanic irritation by the speaking valve and local infections which in turn cause inflammation and edema that leads to ischemia and necrosis. This responds favorably to antiinflammatory drugs and antibiotics. Atrophic enlargement of the fistula however is usually linked to GERD and is harder to treat because it leads to the thinning of the whole posterior tracheal wall [19]. Without regard to the type, fistula enlargement leads to microaspiration of liquids and saliva which progresses towards aspiration of food, spontaneous expulsion or ingestion of the speaking valve, and even pulmonary septic complications.

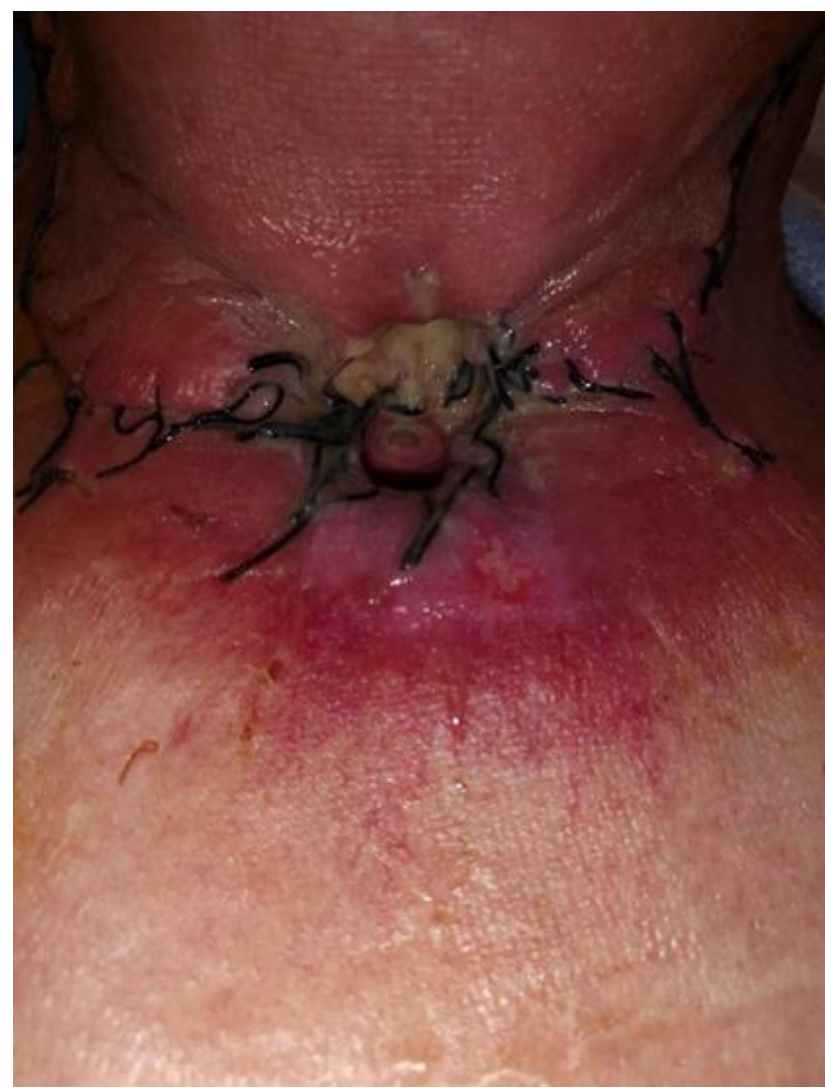

Figure 1 - Total laryngectomy with ,per primam" vocal rehabilitation using tracheoesophageal puncture - day 8 post-op - septicnecrotic enlargement of the fistula (as described by Lorenz)

As described anteriorly, acid reflux erodes the margins of the tracheo-esophageal fistula with progressive enlargement (Figure 2). Bock et. al. performed biopsies from the tracheoesophageal puncture site in 17 total laryngectomy patients and in 10 out of the 17 patients pepsin was found [20]. However, as recent as 2003, studies of complications after vocal prosthesis implantation did not take into consideration supraesophageal reflux as a possible risk factor for these complications [21] [22].

An ample 6 month study from the University of Ulm, under the coordination of K. Lorenz, performed on 60 total laryngectomy patients who had vocal prostheses, analyzed the $24 \mathrm{~h} \mathrm{pH}-$ metry before and after 6 months of PPI therapy. Results showed that patients with supra- 
esophageal reflux treated with PPI had a 10 times lower risk to develop enlargement of the fistula and the complications that derive from it [23].

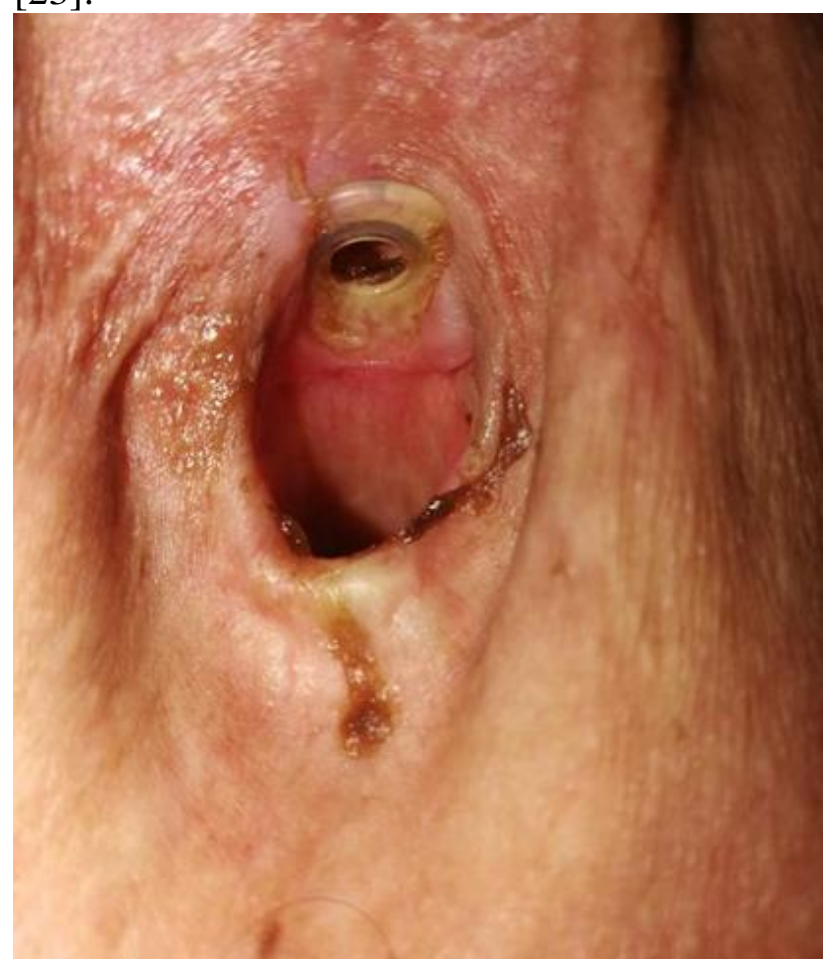

Figure 2 - Chronic periprosthetic inflammation and atrophy of the tracheo-esophageal fistula in a patient with symptoms suggestive of microaspiration (chronic cough - mostly while drinking)

Similar results were reported by the teams led by Cocuzza [24] and Pattani [25] the difference being that this paper took into consideration radiotherapy - which was demonstrated to be another independent risk factor in developing complications of tracheoesophageal speech rehabilitation.

Although generally recognized as an important factor in the failure of vocal rehabilitation by TEP, pepsin levels detected by ELISA in the saliva of laryngectomies by a team led by Hadzibegovic [26] did not correlate with complications of TEP.

\section{Discussion}

Until present day, a consensus on the recommendations for screening and diagnosis of GERD in total laryngectomy patients does not exist. Most studies use $24 \mathrm{~h}$ ambulatory $\mathrm{pH}$ monitoring as a diagnostic tool. Although not a routine test, it's minimally invasive nature and high sensibility recommend it as a viable diagnostic tool.

Prophylactic treatment of GERD after total laryngectomy with $\mathrm{H} 2$ blockers and prokinetics has been demonstrated to reduce the risk of fistula formation - and therefore improves outcome of primary speaking valve placement. There is no data referring to the recommended duration of this treatment.

The decision resides with the ENT physician and should be taken on a per patient basis. It should take into consideration that reflux cannot be prevented in this type of patient and therefore reducing the acidity (by means of PPI therapy) of the secretions has a better therapeutic outcome than other medications. The local examination when changing the speaking valve as well as patient symptoms and complications arisen should prompt the physician to screen for GERD and initiate therapy.

The authors hope that this review of available literature concerning the relationship between supraesophageal acid reflux and the enlarging of the tracheo-esophageal puncture site provides a clear decision tree and sufficient evidence for ENT surgeons to actively search for and treat GERD in their patients, thus permitting better outcomes after voice rehabilitation with TEP.

\section{Conclusions}

All studies show that total laryngectomy patients have a higher susceptibility to GERD, because of mechanic, dynamic and iatrogenic factors. This leads to a higher prevalence of GERD in patients that are at risk to develop local complications, like postoperative pharyngo-cutaneous fistulae. In the subpopulation of laryngectomies that have speaking valves implanted in an iatrogenic tracheo-esophageal fistula, GERD-provoked lesions lead to enlargement of the fistula diameter. This will lead to complications progressing from microaspiration of saliva and liquids to spontaneous expulsion of the vocal prosthesis.

These complications will in turn affect the patient's quality of life and increase the need for changing to larger and larger diameter prostheses or using augmenting flanges to combat the increasing fistula diameter. These 
measures have increased costs and may lead even to hospitalization of the patient and surgery to recalibrate or even temporarily close the fistula.

\section{References}

[1]N. Vakil et al., "The Montreal definition and classification of gastroesophageal reflux disease: A global evidence-based consensus," American Journal of Gastroenterology, vol. 101, no. 8. pp. 1900-1920, Aug-2006.

[2]V. Stanghellini, "Relationship between upper gastrointestinal symptoms and lifestyle, psychosocial factors and comorbidity in the general population: results from the Domestic/International Gastroenterology Surveillance Study (DIGEST)," Scand J Gastroenterol Suppl, vol. 231, no. 23, pp. 29-37, 1999.

[3]E. Cohen et al., "GERD symptoms in the general population: prevalence and severity versus careseeking patients.” Dig. Dis. Sci., vol. 59, no. 10, pp. 2488-96, Oct. 2014.

[4] J. E. Freije, T. W. Beatty, B. H. Campbell, B. T. Woodson, C. J. Schultz, and R. J. Toohill, "Carcinoma of the larynx in patients with gastroesophageal reflux.," Am. J. Otolaryngol., vol. 17, no. 6, pp. 386-90, 1996.

[5]C. A. Gutschow, M. Bludau, D. Vallböhmer, W. Schröder, E. Bollschweiler, and A. H. Hölscher, 'NERD, GERD, and Barrett's esophagus: Role of acid and non-acid reflux revisited with combined pH-impedance monitoring," Dig. Dis. Sci., vol. 53, no. 12, pp. 3076-3081, Dec. 2008.

[6]C. F. Smit et al., "High incidence of gastropharyngeal and gastroesophageal reflux after total laryngectomy," Head Neck, vol. 20, no. 7, pp. 619-622, 1998.

[7]B. LeBlanc, E. Lewis, G. Caldito, and C.-A. O. Nathan, "Increased Pharyngeal Reflux in Patients Treated for Laryngeal Cancer," Otolaryngol. Neck Surg., vol. 153, no. 5, pp. 791-794, 2015.

[8] M. F. Vaezi, S. Singh, and J. E. Richter, "Role of acid and duodenogastric reflux in esophageal mucosal injury: A review of animal and human studies," Gastroenterology, vol. 108, no. 6, pp. 1897-1907, 1995.

[9] J. R. Starling and A. A. Vandemark, "Treatment of hiatal hernia with esophagitis in a patient using esophageal speech after laryngectomy," Am. Surg., vol. 45, no. 6, pp. 407-409, 1979.

[10] J. G. Mathis, G. A. Lehman, J. C. Shanks, E. D. Blom, and R. L. Brunelle, "Effect of gastroesophageal reflux on esophageal speech," J. Clin. Gastroenterol., vol. 5, pp. 503-507, 1983.
[11] H. Maier, "New perspectives on prevention and treatment of complications of speech fistulas," HNO, vol. 58, no. 9, pp. 917-918, 2010.

[12] H. Seikaly and P. Park, "Gastroesophageal reflux prophylaxis decreases the incidence of pharyngocutaneous fistula after total laryngectomy," Laryngoscope, vol. 105, no. 11, pp. 1220-1222, 1995.

[13] R. L. A. Sarría Echegaray P, Tomás Barberán M, Mas Mercant S, Soler Vilarrasa R, "Pharmacological prophylaxis of gastroesophageal reflux. Incidence of pharyngocutaneous fistula after total laryngectomy." Acta Otorrinolaringol Esp, vol. 51, no. 3, pp. 239-42, 2000.

[14] G. Mannelli, R. Santoro, F. Segala, E. Surrenti, and O. Gallo, "Gastro-pharyngeal reflux and total laryngectomy. Increasing knowledge about its management," Am. J. Otolaryngol. - Head Neck Med. Surg., vol. 39, no. 2, pp. 127-132, Mar. 2018. [15] A. İmre, "Complications of tracheoesophageal puncture and speech valves: retrospective analysis of 47 patients," Turkish J. Ear Nose Throat, vol. 23, no. 1, pp. 15-20, Mar. 2013.

[16] T. Malik, I. Bruce, and J. Cherry, "Surgical complications of tracheo-oesophageal puncture and speech valves," Curr. Opin. Otolaryngol. Head Neck Surg., vol. 15, no. 2, pp. 117-122, Apr. 2007.

[17] B. M. Op de Coul, F. J. Hilgers, A. J. Balm, I. B. Tan, F. J. van den Hoogen, and H. van Tinteren, "A decade of postlaryngectomy vocal rehabilitation in 318 patients: a single Institution's experience with consistent application of provox indwelling voice prostheses.," Arch. Otolaryngol. Head. Neck Surg., vol. 126, no. 11, pp. 1320-8, Nov. 2000.

[18] K. J. Lorenz, L. Grieser, T. Ehrhart, and H. Maier, "The management of periprosthetic leakage in the presence of supra-oesophageal reflux after prosthetic voice rehabilitation," Eur. Arch. OtoRhino-Laryngology, vol. 268, no. 5, pp. 695-702, 2011.

[19] A. Rosen, N. Scher, and W. R. Panje, "Surgical Closure of Persisting Failed Tracheoesophageal Voice Fistula," Ann. Otol. Rhinol. Laryngol., vol. 106, no. 9, pp. 775-778, Sep. 1997.

[20] J. M. Bock et al., "Analysis of pepsin in tracheoesophageal puncture sites," Ann. Otol. Rhinol. Laryngol., vol. 119, no. 12, pp. 799-805, 2010.

[21] M. A. Hotz, A. Baumann, I. Schaller, and P. Zbären, "Success and Predictability of Provox Prosthesis Voice Rehabilitation," Arch. Otolaryngol. Neck Surg., vol. 128, no. 6, p. 687, Jun. 2002.

[22] F. W. Stafford, "Current indications and complications of tracheoesophageal puncture for voice restoration after laryngectomy." Curr. Opin. Otolaryngol. Head Neck Surg., vol. 11, no. 2, pp. 89-95, 2003. 
[23] K. J. Lorenz, L. Grieser, T. Ehrhart, and H. Maier, "Role of reflux in tracheoesophageal fistula problems after laryngectomy," Ann. Otol. Rhinol. Laryngol., vol. 119, no. 11, pp. 719-728, 2010.

[24] S. Cocuzza, M. Bonfiglio, R. Chiaramonte, and A. Serra, "Relationship between radiotherapy and gastroesophageal reflux disease in causing tracheoesophageal voice rehabilitation failure," J. Voice, vol. 28, no. 2, pp. 245-249, 2014.
[25] K. M. Pattani, M. Morgan, and C. A. O. Nathan, "Reflux as a cause of tracheoesophageal puncture failure," Laryngoscope, vol. 119, no. 1, pp. 121-125, Jan. 2009.

[26] A. D. Hadzibegovic, D. Danic, D. Prgomet, R. Ticac, and A. Kozmar, "Analysis of Saliva Pepsin Level in Patients with Tracheoesophageal Fistula and Voice Prosthesis Complications," Coll. Antropol., vol. 36, no. 2, pp. 93-97, 2012. 